\title{
Energy Release and Transport Processes in the Centres of Galaxies
}

\author{
Roger Blandford \\ 130-33 Caltech, Pasadena, CA 91125, USA
}

\begin{abstract}
Observations over the past decade have verified, beyond reasonable doubt, that most galactic nuclei contain massive black holes. Hole masses are being measured and firm evidence for spin is being sought. Attention is now returning to the study of how gas flows around black holes and how energy is released both from the accreting gas and from the hole itself in the form of radiation, relativistic jets and non-relativistic, hydromagnetic winds. Some of the different possibilities currently under investigation are briefly reviewed and some recent clues from radio scintillation, polarization and X-ray observations are discussed. It is argued that observations of persistent circular polarisation in Sgr $\mathrm{A}^{*}$ support the presence of an ordered disk magnetic field. It is also conjectured that adiabatic, sub- and super-critical accretion flows are demand-limited, not supply-driven and are associated with large mass outflow as appears to be the case in Sgr $\mathrm{A}^{*}$. This principle may have to be modified when a massive black hole forms in a protogalaxy and this modification may account for the proposed hole mass-bulge velocity dispersion relation. The final stages of this process may release sufficient wind energy from the nucleus to prevent disk formation.
\end{abstract}

\section{Introduction}

This is an exciting time in the study of active galactic nuclei. Radio, optical and $\mathrm{X}$-ray observations are not only persuading the last doubters that most normal galaxies contain massive black holes, but are measuring of their masses [Dressel, Baan, Greenhill] $]^{1}$ and probing their spins. Furthermore, the high angular resolution accessible to ground-based and space-based observation of relativistic jets is allowing us to trace these outflows down to radii less than $\sim 100 \mathrm{~m}$ vindicating the view that they are at least powered by physical processes located fairly close to the central black hole [Falcke, Hirabayashi]. Finally, radio polarimetric and $\gamma$-ray [Edwards] observations of these relativistic jets are being interpreted in a manner that allows us to make arguments concerning their composition, which, in turn, impact our understanding of how they are formed. Many of these topics are described at greater length elsewhere in these proceedings. I shall just emphasize some more recent theoretical ideas.

\footnotetext{
${ }^{1}$ References in square brackets refer to other papers in these proceedings.
} 


\section{Accretion Power vs Black Hole Power}

As is often the case, the most important factor controlling the evolution of accretion disks is the flow of energy. There are two power sources. Firstly, accreting gas will release its binding energy before it makes its final adiabatic plunge towards the event horizon. However, the energy will generally not be liberated all at the point of release because the magnetic torques which transport angular momentum, also transport energy outward radially through the disk. (There can be additional, convective and radiative energy transport which is ineffective in transporting angular momentum.)

There are several possible channels through which this energy can escape and their relative importance surely depends upon the accretion rate. It can be radiated from a disk photosphere. It can emerge from the disk surface as a hydromagnetic energy flux and heat an active corona, which can in turn radiate non-thermally (for example by Comptonising disk photons) or drive a wind. The coronal heating will probably occur via magnetic reconnection or a turbulent cascade. Energy and angular momentum can also be extracted from the disk if there is a large scale field that launches a hydromagnetic wind. There will then be an external torque per unit radius $d G / d r$ which will extract energy at a rate $\int d G \Omega$ where $\Omega$ is the disk angular velocity without dissipation.

The second source of energy is the spin of the hole. As is well known, up to 29 percent of the mass of a spinning black hole is, in principle, extractable (eg Lee, Wijers \& Brown 1999). This is $6 \times 10^{62}\left(M / 10^{9} \mathrm{M}_{\odot}\right)$ erg. In practice no more than, say, a quarter of this energy is likely to be available, but still it is ample to account for the non-thermal emission associated with the most profligate quasars. It is important to realize that, although energy is not strictly localisable in general relativity, the spin energy is just as much associated with the so-called ergosphere outside the event horizon as with the interior of the hole. (The ergosphere can be defined as the region within which a distant observer must rotate with respect to the non-rotating frame at infinity.) This is a crucial and important difference from a spinning, newtonian star.

Again there are many possibilities [Meier]. It is possible to extract work from orbiting matter which can be dragged by magnetic stress on to lower or even negative energy (including rest mass) orbits within the ergosphere (e. g. Ruffini \& Wilson 1975; Agol \& Krolik 2000). When this matter enters the horizon, the net gravitational mass of the hole will increase by less than the accreted rest mass and will actually decrease if the orbit has negative energy. If the matter is attached to open magnetic field lines, then work is done on the base of the jet and drives an outflow, either continuously or intermittently. Alternatively, if the infalling matter is magnetically attached to the disk and still in causal contact, that is to say outside the relevant, ingoing critical points, then orbital energy and angular momentum can be transferred to the disk from within its innermost stable circular orbit, thereby enhancing the disk power. In either case, it is an arbitrary choice whether to charge the energy extracted to the hole or the accreting matter. Magnetic flux that threads the horizon can transfer energy directly from the hole to the jet or the disk at a rate $\sim a^{2} B^{2} c$, where $a$ is the specific angular momentum of the hole and $B$ is the field strength. To an observer hovering just above the horizon and, necessarily, orbiting with the hole angular velocity, the electromagnetic energy flux will appear to enter the hole. 
However energy flux is conserved in the non-rotating frame and transforming into this frame produces an outward energy flux at the horizon. In other words, the black hole, a spinning conductor, acts like a giant battery. Under the conditions invoked for Cygnus A, say, an EMF of $\sim 10^{20} \mathrm{~V}$ is induced, with a field $B \sim$ $10^{4} \mathrm{G}$, and the current is $\sim 10^{18} \mathrm{~A}$. (The resistance $\sim 100 \Omega$ turns out to be similar to the impedance of free space. The merit of this process as a candidate for powering relativistic jets is that the electromagnetic power is likely to be relatively uncontaminated with baryons, the disadvantage is that if the field strength above the hole is comparable with that above the inner disk then, the relatively small area of the horizon will ensure that the hole power be less than the disk power, which, although adequate for most jets may not be sufficient for the most luminous examples. Note, though, that this conclusion does not follow if, as commonly envisaged, the disks associated with powerful radio galaxies are thick.

There is actually a second way to extract energy from the hole. If the disk plane wobbles about the equatorial plane of the hole, then there will be a "Bardeen-Petterson" torque that acts between the disk and the hole. The nature of this torque has been quite controversial over the years, but the net result is that the disk will be brought into alignment with the hole within some radius, $r_{\mathrm{BP}}$. There will be a reaction torque operating on the hole and an associated dissipation in the disk near $r_{\mathrm{BP}}$. This could provide a significant local heating of the disk under some models (eg Natarajan \& Pringle 1998)

\section{Jets}

So far, I have emphasized the (electro)magnetic extraction of energy from the disk and the hole because most models are now of this type. However, this does not imply that observed jets mostly carry Poynting flux. Just as is the case with the Crab Nebula, there is believed to be a transformation of the energy flux from mechanical to electromagnetic to particle. The issue now is where and how do these transitions occur. One extreme possibility is that a hot plasma forms through magnetic dissipation in the corona itself and that this flows hydrodynamically into the jet. (This would probably have to be an electron-ion plasmas as it is very hard to see how a pair plasma could be accelerated to ultrarelativistic bulk speed close to the hole without suffering catastrophic radiative drag.) Another possibility is that ultrarelativistic ions are formed close to the hole and somehow directed along a jet and that these cascade down to lower energy particles that will produce the radiation we observe. In many regards the most natural scheme is that the energy is carried away electromagnetically from the vicinity of the hole and that the pair fraction gradually builds up at a rate that may be limited by annihilation. Provided that this happens after the radiative drag is ignorable, an ultrarelativistic jet will ensue.

Extracting the energy is only half of the challenge in forming a jet. The outflow must also be tightly collimated. For a long while it has been thought that jets required disks. Howevever, with the discovery of jets associated with the Crab and Vela pulsars, perhaps this is not even necessary. Magnetic collimation is most commonly invoked simply because it is hard to sustain the necessary gas pressure against large radiative loss at the location where the collimation 
must be occuring. (Note that this does not imply that gas pressure is irrelevant. Magnetic stress must be ultimately confined transversely at large cylindrical radius and static gas pressure or ram pressure is surely responsible.)

There are still choices that have to be made in magnetic collimation. The confining stress can be attributed to the pressure of poloidal magnetic field or the tension associated with the toroidal field. Alternatively, the field may be quite disorganised but show an anisotropic stress tensor which somehow achieves the necessary collimation.

One argument that has often been made against magnetic collimation, particularly by toroidal field is that such configurations are probably unstable. However, this may not be such an unwelcome conclusion because observed jets often do appear to corkscrew in real space and this is a natural instability mode for a pinched jet. At an even more extreme level, many jets appear to be episodic and to exhibit strong internal shocks [Klare]. This, too, can be regarded as the nonlinear development of a jet instability. One possibility is that the natural increase in the ratio of the toroidal to the poloidal field with distance along the jet is balanced by steady magnetic reconnection, which limits the growth of toroidal field and lead to the steady acceleration of electrons and positrons.

In order to construct more quantitative models of jets, it is necessary to make better estimates of the power that they carry (as distinct from the power that they radiate). There are several approaches that have been followed. One is to assume that the pressure is well-estimated by the equipartition pressure and that the jet expands on the Mach cone. In this case the power will be $L_{\mathrm{j}} \sim P_{\mathrm{eq}} r^{2} V_{\mathrm{j}}$, a formula that is roughly valid relativistically. Alternatively, if the external pressure is known and the jet is thought to be in pressure equilibriium with its surroundings, then we can replace the equipartition pressure with the external pressure. A third approach is to estimate the power supply to large double lobes. Using arguments like this it has been possible to argue that the jet power in some sources, including M87 [Junor] and some blazars, exceeds the bolometric power. If this were generally true, then it would effectively rule out many jet formation models. The mid infrared observations of SIRTF will be crucial for measuring the total nuclear luminosity of radio galaxies.

\section{Brightness Temperature and Polarisation}

One of the more interesting and potentially significant developments in recent years has been the accumulation of evidence for high brightness temperatures in compact extragalactic radio sources. Intraday variable sources exhibit measured brightness temperatures that are at least as high as $\sim 3 \times 10^{13} \mathrm{~K}$ and could be significantly larger [Jauncey, Dennett-Thorpe, Kedziora-Chudczer]. This is conventionally interpreted in terms of the traditional inverse Compton limit. For the observed ratios of Compton to synchrotron power in jets, the comoving brightness temperature has an upper limit of $\sim 10^{12} \mathrm{~K}$. One power of the Doppler factor, which is twice the relevant Lorentz factor converts to the comoving frame suggesting that $\Gamma>20$. (Large Lorentz factors are not so intimidating now as values of $\Gamma \sim 300$ are derived for $\gamma$-ray bursts although the radiative efficiency may be low at radio wavelengths.) If it turns out that comoving brightness temperatures well in excess of the Compton limit are required then it may be 
necessary to invoke a coherent emission mechanism. This is not, by itself, unreasonable; after all, the Sun and Jupiter support coherent emission under relatively benign conditions. A cyclotron maser is a particularly enticing possibility. What may be a bit more problematic is the escape of very high brightness radiation from the source when it is subject to nonlinear induced Compton and Raman scattering (Levinson \& Blandford 1995).

Now, in a self-absorbed synchrotron source, close to the inverse Compton limit, the energies of the emitting electrons at the radio photosphere must be $\sim 300 \mathrm{MeV}$. We do not see the emission from lower energy electrons and it is interesting to ask if they are present and if the electrons that we do see have proton or positron partners. As argued by Wardle et al. (1998 and references cited therein), in the former case, the lower cut off in the electron distribution function must be $\sim 50 \mathrm{MeV}$, otherwise there will be too much jet kinetic energy, carried mainly by the protons. In the latter case, the pair distribution function will extend down to mildly relativistic energy Similar conclusions are drawn from the observation of strong linear polarization which limits the internal Faraday rotation within the source. Finally the detection of persistent circular polarisation [Macquart] has been used to rule in favor of a pair plasma, on the grounds that the frequency dependence is better fit by Faraday conversion in a pair plasma than intrinsic circular polarisation in a proton plasma. Note that if the radiation mechanism is coherent cyclotron radiation, then the fact that the polarisation is not even stronger may be an indication that there is a pair plasma present.

\section{Galactic Centre}

The 2.6 million solar mass black hole at our Galactic center is in many respects the best example to study because it is so inactive [Eckart]. It has recently been detected in X-rays with a very soft 2-10 keV luminosity (Baganoff 2000) of $\sim 2 \times 10^{33} \mathrm{erg} \mathrm{s}^{-1}$, which may be variable. This is a strikingly low luminosity. The independently estimated mass supply rate is very roughly $\sim 10^{22} \mathrm{~g} \mathrm{~s}^{-1}$ and yet the bolometric luminosity is $\sim 10^{36} \mathrm{erg} \mathrm{s}^{-1}$ corresponding to an apparent radiative efficiency $\sim 10^{-7} c^{2}$ [Coker]. As has been discussed elsewhere by Blandford \& Begelman (1999), this is consistent with the theoretical prediction that adiabatic (in the sense that there is no loss of heat) accretion flows lose a large fraction of their mass before crossing the horizon. This is in contrast to the prediction of the widely-discussed and conservative ADAF models. (A naive interpretation of the $\mathrm{X}$-ray observations suggests a maximum mass accretion rate of $\sim 10^{20}\left(T_{e} / 10^{10} \mathrm{~K}\right)^{1 / 4} \mathrm{~g} \mathrm{~s}^{-1}$. It could plausibly be much lower.) The reported spectrum suggests that the X-ray emission is nonthermal, which is really expected, because it would be remarkable if trans-sonic, trans-Alfvénic, trans-relativistic flows avoided accelerating relativistic particles.

If the mass accretion rate really is very low, then this opens up the possibility that we may be seeing radio or, more likely, $\mathrm{mm}$ emission from very close to the hole and that polarization observations might be quite diagnostic of the physical conditions. Now, in the case of Sgr $\mathrm{A}^{*}$, there is no linear polarization reported at radio wavelengths (although it has been reported, quite surprisingly, at submm wavelengths). The lack of linear polarization is quite 
consistent with the high Faraday rotation expected from an accretion flow onto a black hole. However, variable circular polarization has been reported with a persistent sign. There is the intriguing possibility that this reflects the orbital motion of the plasma around the central black hole. To see how this might come about, consider a magnetoactive plasma with $X=\omega_{P}^{2} / \omega^{2}, Y=\omega_{G} / \omega<1$. Under quasi-longitudinal conditions, essentially $\cos \theta<Y$, where $\theta$ is the angle between $\vec{k}$ and $\vec{B}$, the electromagnetic eigenmodes are elliptically polarized with axis ratio $r=1 \pm Y \sin \theta \tan \theta$ and phase velocity difference $\Delta V=c X Y \cos \theta$.

Now consider synchrotron or cyclotron radiation emitted from within an accretion disk of thickness $H$. The major axis of the polarization ellipse will be Faraday rotated at a rate $\Delta \Phi / d s=\Delta V \omega / 2 c^{2}$. Now, we expect that the magnetic field will reverse often along a ray and as $X|Y| \omega H / c$ is anticipated to be much greater than unity, then we expect that the emergent linear polarisation will be vanishingly small. (The limiting polarisation along an individual ray is likely to be determined by the decrease in the density rather than the magnetic field strength.)

The circular polarisation is a bit more problematic. If we suppose that there is a net magnetic field normal to the disk, as is true of some models, then there should be a preferred sense of the circular polarization that is emitted in cyclotron or low energy synchrotron radiation. This will be largely preserved in propagating out of the disk. However, eventually, the sign of the net vertical field must change and we might even be able to see this change propagate from low to high frequency if, as seems reasonable, there is a radius to frequency mapping in the disk. However, it is also possible that there is essentially no large scale field. In this case, there is a second mechanism which may create circular polarisation.

Consider a single eigenmode propagating out of the disk through a spatially varying magnetic field. We suppose that the variation happens relatively slowly on the scale of the wavelength so that the polarization adjusts adiabatically (with no mode crossings). We ignore refraction and recall that the electric vector is normal to $\vec{k}$. Next, suppose that the two eigenmodes are launched with equal amplitude and that the field is uniform. The beating between the two eigenmodes will result in a circular polarisation of amplitude $Y \sin \theta \tan \theta$ that changes sign as the plane of linear polarisation rotates. The limiting circular polarisation that emerges from the disk is equally likely to have either sign and so there will be no net circular polarisation. Now let the magnetic field direction vary along a ray. If the angle $\theta$ varies, then there will be a corresponding change in the axis ratio of the polarization ellipse, but still no preference for one sign over the other. However, if the azimuthal angle $\phi$ relating $\vec{B}$ to $\vec{k}$ changes in a systematic fashion along all rays then it appears that the circular polarisation will have one sign as opposed to the other for slightly more than half the time. The average circular polarization along a ray will be $\sim<Y \sin \theta \tan \theta d \phi / d \Phi>\sim$ $|Y \ln Y| d \phi / d \Phi$.

Now this is just what we might anticipate in a magnetised accretion disk. In the disk interior, the typical field direction will trail to reflect the differential rotation in the disk. However, the field will be swept back by progressively smaller angles as the ray approaches the disk surface, corresponding to a net rotation of the average azimuthal angle $\phi$. There will only be a preferred sense 
of limiting circular polarization, if the magnetostatic field is still changing in this systematic manner over the last radian of Faraday rotation. The net circular polarization will be $\sim c \ln Y / \omega H X$, where $H$ is the disk thickness. However, if $X>10^{-6}$, as seems assured in $\mathrm{Sgr} \mathrm{A}^{*}$, then this mechanism is unlikely to create the observed circular polarisation at radio wavelengths, although it may be relevant in the sub $\mathrm{mm}$. I conclude that the measurement of persistent circular polarization is more naturally interpreted in terms of a long term, ordered magnetic field, as discussed above. There may be a related effect that can occur in the ergosphere, where the dragging of inertial frames is reponsible for a rotation of the polarisation ellipse. These ideas are currently under study.

\section{Black Hole Formation}

The foregoing considerations apply to sub-critical accretion. Similar principles are relevant to super-critical accretion. Here the problem is not creating the photons, but allowing them to escape. Electron scattering opacity almost certainly dominates the radiative transfer and the photons will be trapped out to a radius $r_{\mathrm{tr}}=\dot{M} \kappa_{T} / 4 \pi c$. Within this radius, we can regard the inflow as adiabatic, with an outward transport of energy and angular momentum. I have conjectured elsewhere that supercritical accretion is "demand-limited" rather than "supply-driven". In other words black holes only grow at roughly the critical rate, $\dot{M}=4 \pi G M / \epsilon \kappa_{T} c$, where $\epsilon \sim 0.1$ is the overall efficiency of energy release. If a black hole is supplied at a greater rate, it will radiate at a few times the Eddington luminosity and the excess mass and energy will be carried off in an outflow with a similar power. The mass may be liberated at all radii so that the local net mass accretion rate grows roughly linearly with radius. Alternatively, there could be strong, internal circulation which carries the energy released near the black hole to some outer radius from where the wind is launched. This behaviour is, arguably, observed in Broad Absorption Line Quasars and some of the most luminous, Galactic, transient sources [Spencer].

If this principle were correct, it would raise an important question. How do black holes grow to a billion solar masses, when the universe was less than $\sim 1$ Gyr old (as observations of high redshift quasars now suggest) if there were only time for a few e-foldings in mass? One possible answer is that large holes might have formed by dynamical collapse. However, this seems a bit unlikely as they should have made a sort of mega gamma ray burst, and there is no evidence for such prolonged, luminous explosions on our past light cone. Alternatively, what may be different about accretion during the early epochs of galaxy formation is that the black hole should be surrounded by a chaotic, self-gravitating reservoir of gas, extending from the Bondi radius, $r_{\mathrm{B}} \sim G M / \sigma^{2}$ where $\sigma$ is the velocity dispersion determined by the dark matter, stars and gas to some outer radius, $r_{\text {gal }}$. We expect the gas initially to dominate the mass in the inner parts of the galaxy.

Now, modifying a suggestion of Silk \& Rees (1998), suppose that a small black hole forms. The maximum, net mass supply rate is the Bondi (or Jeans') rate, $\dot{M} \sim \sigma^{3} / G$ under these conditions. If this were to accrete onto the black hole and release energy efficiently, the resulting wind would quickly blow away all the surrounding gas and shut off the supply. What seems most likely 
is that the feedback limits the accretion rate to a value such that the wind power is just sufficient to drive away the accreting gas, ie to $\dot{M} \sim \sigma^{5} / \epsilon G c^{2} \sim$ $2 \times 10^{-3}\left(\sigma / 100 \mathrm{kms}^{-1}\right)^{5} \mathrm{M}_{\odot} \mathrm{yr}^{-1}$. The hole grows until it has a mass $M \sim$ $2 \times 10^{5}\left(\sigma / 100 \mathrm{kms}^{-1}\right)^{5} \quad \mathrm{M}_{\odot}$ after which point the accretion rate will probably be Eddington-limited and the hole mass will grow exponentially until the wind associated with a sub-critical accretion (formed with an efficiency $\epsilon_{W}$ ) can limit the accretion again. If we allow this phase to continue for a dynamical time, $\sim 3 r_{\text {gal }} / \sigma$ then the final black hole mass will be $M \sim 3 \sigma^{4} r_{\text {gal }} / \epsilon_{W} G c^{2} \sim$ $6 \times 10^{6}\left(\sigma / 100 \mathrm{kms}^{-1}\right)^{4}\left(r_{\text {gal }} / 10 \mathrm{kpc}\right)\left(\epsilon_{W} / 10^{-3}\right)^{-1} \mathrm{M}_{\odot}$. Choosing plausible values $\epsilon_{W} \sim 10^{-3} ; r_{\text {gal }} \sim 10 \mathrm{kpc}$ give a relation similar to that found by Ferrarese et al. (2000) and Gebhardt et al. (2000), [Marconi, van der Marel].

Obviously, this is a gross over-simplification of the many complex processes at work, simultaneously, in a nascent galaxy. Radiative cooling and star formation will be stimulated in the surrounding gas and this may cause additional heating. The direct and indirect influence of the hole may soften the potential. Rotation and mergers are surely relevant. Most importantly, I have not specified whether or not the gas falls in towards the nucleus or moves out towards $r_{\text {gal }}$ whether galaxies form from the outside in or the inside out. This raises a radical possibility. Perhaps early-type galaxies are so called because their central black holes grow quickly and expel enough gas to prevent the subsequent formation of disks. This scheme, which is subject to hydrodynamic simulation, has clear, observable implications.

Acknowledgments. I am indebted to many collaborators, including Avery Broderick, Marco Salvati, Todd Small and especially Mitch Begelman for discussion of the above. Support under NSF grant AST 99-00866 and NASA grant 5-2837 is gratefully acknowledged.

\section{References}

Agol. E. \& Krolik, J. 2000, ApJ, 528, 161

Baganoff, F. et al. 2000 Paper presented at November 2000 HEAD meeting, Hawaii

Blandford, R. D. \& Begelman, M. C. 1999, MNRAS, 303, L1

Ferrarese, L. \& Merritt, D. 2000, ApJ, 539, L9

Gebhardt, K. et al. 2000 ApJ, 539, L13

Lee, H-K, Wijers, R. A. M. J. \& Brown, G. E. 1999, Phys. Rep., 325, 83

Levinson, A. \& Blandford, R. D. 1995, MNRAS, 274, 717

Nandra, P. 2000 Proc. X-ray Astronomy '99 - Stellar Endpoints, AGN and the Diffuse Background, in press

Natarajan, P. \& Pringle, J. 1998, ApJ, 506, L97

Ruffini, R. \& Wilson, J. R. 1975, Phys.Rev.D, 12, 2959

Silk, J. \& Rees, M. 1998, A\&A, 331, L1

Wardle, J. F. C. et al. 1998, Nature, 395, 457

Wehrle, A. et al. 1998, ApJ, 497, 178

Wilson, A., Shopbell, P. \& Young, A. 2000, ApJ, in press 